1 Secretaria Municipal de Saúde de Canaã dos Carajás - Canaã dos Carajás (PA), Brasil. gutenf@yahoo.com.br

2 Universidade do Estado do Pará (Uepa), Departamento de Ciências do Movimento - Belém (PA), Brasil.

rodrigolsf@yahoo.com.br

3 Universidade Federal do Pará (UFPA) - Belém (PA), Brasil.

mariliabrasil@terra.com.br

\section{Hanseníase e Atenção Primária à Saúde: uma avaliação de estrutura do programa}

\author{
Leprosy and Primary Health Care: program structure assessment
}

Gutembergue Santos de Sousa', Rodrigo Luis Ferreira da Silva², Marília Brasil Xavier ${ }^{\mathbf{3}}$

RESUMO O presente trabalho objetivou avaliar a estrutura do programa municipal de controle da hanseníase, em Canaã dos Carajás (Pará), no contexto da Atenção Primária à Saúde. Tratase de um estudo de avaliação de programas de saúde, com foco na hanseníase, tendo como público-alvo os gestores do programa de hanseníase e os gerentes das unidades de saúde da atenção primária. Foram utilizados dois questionários diferentes, elaborados especificamente para esse fim. Concluiu-se que o município em questão possui uma estrutura classificada entre insatisfatória e regular, demonstrando várias fragilidades no programa avaliado, para o atendimento em hanseníase.

PALAVRAS-CHAVE Hanseníase. Avaliação em saúde. Estrutura dos serviços. Atenção Primária à Saúde. Avaliação de serviços de saúde.

\begin{abstract}
Objective: to evaluate the structure of the municipal program of leprosy control in Canaã dos Carajás (Pará), in the context of Primary Health Care. Methods: this is an assessment of health programs, focusing on leprosy, targeting managers of the leprosy program and managers of health facilities of primary care. Two different questionnaires, developed specifically for this purpose, were used. Conclusion: the municipality in question has a structure classified between poor and regular, showing several weaknesses in the assessed program for the care of leprosy.
\end{abstract}

KEYWORDS Leprosy. Health evaluation. Structure of services. Primary Health Care. Health services evaluation. 


\section{Introdução}

A hanseníase é uma doença infecciosa e crônica de grande relevância para a saúde pública, possuindo ações exclusivas voltadas para a sua eliminação em âmbito nacional por meio do Programa de Controle da Hanseníase, presente na Atenção Primária à Saúde (APS), em particular nas Equipes de Saúde da Família (EqSF), atendendo a população por meio de ações preventivas e curativas (BRASIL, 2007). É transmitida pelo contato direto com pessoas doentes, principalmente mediante o convívio com pacientes multibacilares antes do tratamento, com interferência de fatores determinantes e condicionantes do meio em que vive (SARNO, 2003).

Somente no ano de 2012, foram notificados 33.741 mil casos novos da doença no País, com uma taxa de incidência de 17,39 por 100 mil habitantes. No estado do Pará, em 2012, foram notificados 3.970 novos casos de hanseníase, com uma taxa de incidência de 50,75 por 100 mil habitantes. No município de Canaã dos Carajás (PA), durante o ano de 2012, foram notificados 22 novos casos, com uma taxa de incidência de 75,60 por 100 mil habitantes (BRASIL, 2012). Os dados acima apresentados fazem com que a hanseníase seja considerada um sério problema de saúde pública, exigindo prioridades nas políticas públicas e ações de saúde que quebrem a cadeia de transmissão e que, consequentemente, cause impacto nos coeficientes da doença.

A incidência da hanseníase no município em questão é quatro vezes superior à média da taxa apresentada no Brasil no mesmo período, e bem maior do que os dados apresentados no estado do Pará, fazendo com que o município seja hiperendêmico em relação à hanseníase, alertando para a necessidade de intervenção no local e de um melhor conhecimento sobre o funcionamento do programa em questão. A alta taxa preocupa tanto as equipes de saúde quanto a gestão, cujos determinantes são potencializados ante o fato de o município possuir um grande número de alojamentos coletivos, áreas de difícil acesso e dificuldade no controle dos comunicantes da doença devido ao grande fluxo migratório.

Avaliar, segundo a Organização Mundial da Saúde (OMS) (2010), é realizar uma análise sistemática do desempenho do programa após um período de tempo específico de sua implementação, comparando os resultados alcançados com os esperados, considerando o planejamento prévio. A avaliação considera aspectos relacionados com a qualidade, eficiência, equidade, relevância, sustentabilidade, qualidade dos cuidados e indicadores de estrutura física.

A avaliação nos serviços de saúde, segundo Ferreira (2005), traduz-se em uma prestação de contas à sociedade sobre as ações e medidas que estão sendo utilizadas no serviço, contribuindo para a melhoria do serviço em questão, com reflexo direto na qualidade de vida da população assistida pelo programa avaliado.

Para Lanza (2014), avaliar a hanseníase é de extrema importância por se tratar de um agravo prioritário na política de saúde do Brasil necessitando de ações que visem ao fortalecimento da atuação da APS no seu controle. $\mathrm{O}$ uso de instrumentos adequados e que permitam a análise do conhecimento dos atributos que estão sendo alcançados facilita o planejamento das ações para o respectivo serviço, repercutindo diretamente na qualidade deste.

Diante do exposto o presente estudo teve como objetivo avaliar a estrutura do programa municipal de controle da hanseníase, em Canaã dos Carajás, no contexto da APS, sob a ótica da gestão e da gerência do programa em questão.

\section{Material e métodos}

Trata-se de um estudo de avaliação em saúde, tendo como foco o programa de hanseníase, buscando um aprofundamento 
acerca das condições estruturais e de funcionamento do respectivo programa em Canaã dos Carajás, que em 2015 possuía uma populacional estimada de 33.632 habitantes (IBGE, 2015). Contudo, o que se observa no município, e pelo discurso de seus gestores, é que tais números não representam a população real residente, tendo em vista a elevada contratação de trabalhadores para atuar na montagem de projeto minerador, acarretando com isso um desordenado processo migratório de pessoas em busca de trabalho.

O caráter flutuante de sua população e a grande taxa de crescimento anual traz consigo uma série de dificuldades para os serviços públicos de saúde, principalmente no que tange ao planejamento das ações e serviços e à implementação de políticas públicas de saúde.

A avaliação de estrutura, segundo Donabedian (1980), contempla análise de instrumentos, recursos (financeiros, físicos e humanos) e lugares físicos (estrutura física e materiais) necessários ao desenvolvimento da atenção à saúde.

Para esta avaliação, foram utilizados dois questionários semiestruturados, composto de perguntas abertas e fechadas. Para Gil (2008), o questionário é uma técnica de investigação social, contendo um conjunto de questões, que serão respondidas pelos sujeitos da pesquisa, com a finalidade de evidenciar informações sobre conhecimentos, valores, interesses, expectativas, comportamento, entre outros, apresentando uma variedade muito grande de temas, de acordo com o problema de pesquisa apresentado e com os objetivos que se pretende alcançar.

Quanto à análise e interpretação dos resultados, este estudo teve uma abordagem quantitativa, dos seguintes aspectos: estrutura física, logística de trabalho, recursos humanos disponíveis, recursos materiais existentes, estratégias utilizadas pelo programa, atividades realizadas e recursos financeiros disponibilizados.

A pesquisa foi realizada com nove EqSF e quatro equipes de Agentes Comunitários de Saúde, além de gestores da Secretaria Municipal de Saúde de Canaã dos Carajás onde foram abordados os assuntos inerentes à gestão do programa. A amostra foi composta por dois gestores e oito gerentes, sendo nove enfermeiros e um administrador.

As informações quantitativas foram apresentadas em tabelas e analisadas por meio de frequências relativas, correspondendo às variáveis presentes nos questionários aplicados.

O estudo foi submetido ao Comitê de Ética em Pesquisa do Núcleo de Medicina Tropical da Universidade Federal do Pará, com parecer favorável $\mathrm{n}^{\circ} 1.128 .385$, foram observados os critérios para o estudo em seres humanos conforme estabelece a Portaria $n^{\circ} 466 / 12$.

\section{Resultados}

O município conta com 10 unidades de APS que fazem atendimento do agravo em questão, sendo seis delas na área urbana e quatro na área rural. Entre os profissionais de saúde que atendem a demandas relacionadas com a hanseníase, o município conta com uma equipe formada por médicos, enfermeiros e fisioterapeutas para atendimento direto ao usuário do programa sem necessidade de referenciar para tais profissionais. O quadro de profissionais do município também possui psicólogos, assistentes sociais, neurologista e ortopedistas alocados em outros setores, mas que atendem a encaminhamentos do programa de acordo com as necessidades.

Conforme relato do gestor do referido programa, o diagnóstico da hanseníase é de responsabilidade das unidades básicas de saúde, porém alguns casos ainda são diagnosticados em outros serviços, tais como hospitais e ambulatório de especialidades. Todas as unidades de saúde do município possuem local adequado para o diagnóstico e atendimento sequencial ao usuário do programa de hanseníase, bem como os 
equipamentos e materiais necessários para a realização do atendimento em questão. A referência do programa funciona por meio de pactuação com o município vizinho, localizado a $62 \mathrm{~km}$ de distância.

O município não possui plano municipal de controle da hanseníase, sendo suas ações baseadas e planejadas de acordo com a programação anual de saúde e planejamentos setoriais das equipes de trabalho.

Foram realizadas uma campanha para detecção de casos novos no ano de 2014 e uma campanha no ano de 2015, sendo que ambas as campanhas estavam previstas e detalhadas na programação anual de saúde do município.

Conforme informações do gestor do programa, é realizado em média um treinamento anual para a equipe de saúde, no manejo clínico da hanseníase, envolvendo os profissionais médicos, enfermeiros, fisioterapeutas e terapeutas ocupacionais.
Não foi relatada dificuldade para aquisição dos materiais e insumos necessários ao desenvolvimento das ações do programa municipal de controle da hanseníase, sendo utilizados recursos próprios nessas aquisições quando necessário, atendendo a todas as solicitações oriundas das equipes de saúde no que tange ao programa.

Não existe uma programação orçamentária financeira, estabelecida por meio de dotação orçamentária, no orçamento anual da secretaria municipal de saúde, específica para as ações de hanseníase, sendo que estas são atendidas dentro do bloco financeiro da vigilância em saúde.

É disponibilizado veículo para a equipe de saúde realizar busca ativa aos faltosos do programa municipal de controle da hanseníase, bem como também é fornecido combustível mensalmente ao enfermeiro da equipe de saúde caso este opte por realizar tais visitas em seu próprio carro.

Tabela 1. Materiais sobre hanseníase disponíveis nas unidades de saúde. Canaã dos Carajás (PA) - 2016

\begin{tabular}{lccr}
\hline \multirow{2}{*}{ Material } & \multicolumn{2}{c}{ Unidades de Saúde Disponíveis } \\
\cline { 2 - 4 } & Sim & Não & Total \\
\hline Manual do Programa de Hanseníase & $100 \%$ & $0 \%$ & $100 \%$ \\
Protocolo do Programa de Hanseníase & $75 \%$ & $25 \%$ & $100 \%$ \\
Protocolo complementar para investigação Diagnóstica em & $50 \%$ & $50 \%$ & $100 \%$ \\
menores de 15 anos & & $37,5 \%$ & $100 \%$ \\
Folder & $62,5 \%$ & $0 \%$ & $100 \%$ \\
Cartazes & $100 \%$ & & \\
\hline
\end{tabular}

Fonte: Elaboração própria a partir dos dados coletados.

A tabela 1 refere-se aos materiais disponíveis nas unidades de saúde da atenção primária, referentes ao programa de hanseníase. Observa-se que $100 \%$ das unidades possuem manual do programa de hanseníase, $75 \%$ possuem o protocolo do programa e apenas $50 \%$ das unidades possuem o protocolo complementar para investigação diagnóstica em menores de 15 anos.

O município não possui protocolo próprio e nem fluxograma detalhado para o atendimento dos casos de hanseníase, sendo que todas as diretrizes utilizadas para atendimento no programa são baseadas no Guia 
de Vigilância Epidemiológica (Ministério da Saúde - MS) e nas portarias do MS que dispõe sobre o agravo em questão.

Quanto à presença de materiais de divulgação e materiais educativos, observa-se que $100 \%$ das unidades possuem cartazes afixados sobre a hanseníase embora apenas $62,5 \%$ das unidades possuam folder para divulgação da doença.

Todas as unidades de saúde visitadas possuem os materiais básicos para a realização do diagnóstico da hanseníase, em um kit específico para essa finalidade, conforme demonstra a tabela 2 .

Tabela 2. Materiais disponíveis nas unidades de saúde para o diagnóstico de hanseníase. Canaã dos Carajás (PA) - 2016

\begin{tabular}{lccr}
\hline \multirow{2}{*}{ Material } & \multicolumn{3}{c}{ Disponibilidade } \\
\cline { 2 - 4 } & Sim & Não & Total \\
\hline Monofilamentos de Semmes Weinstein (kit com 6) & $100 \%$ & $0 \%$ & $100 \%$ \\
Fio dental & $100 \%$ & $0 \%$ & $100 \%$ \\
Algodão & $100 \%$ & $0 \%$ & $100 \%$ \\
Alfinetes & $100 \%$ & $0 \%$ & $100 \%$ \\
Tubo de ensaio & $100 \%$ & $0 \%$ & \\
\hline
\end{tabular}

Fonte: Elaboração própria a partir dos dados coletados.

Referente à realização do teste de acuidade visual no diagnóstico da hanseníase, $75 \%$ das unidades de saúde não realizam tal teste, 12,5\% realizam para todos os pacientes no momento do diagnóstico e $12,5 \%$ realizam, porém sem rotina estabelecida. Quanto aos fatores ligados a não realização do teste acima citado, os depoentes relatam a falta de material necessário e a falta de espaço físico adequado.

A dispensação dos medicamentos da poliquimioterapia da hanseníase e dos medicamentos utilizados para tratamento das reações hansênicas também foram objetos de avaliação deste estudo, que identificou que $50 \%$ das unidades de saúde possuem tais medicamentos em estoque armazenados em sua sede enquanto os demais $50 \%$ não possuem armazenamento, dependendo de liberação conforme notificação de casos novos.

O serviço de saúde usa o cartão de aprazamento das consultas, inclusive para as referências para o serviço especializado.

Entre as ações de monitoramento implementadas em pacientes em uso de prednisona, $100 \%$ das unidades de saúde realizam peso e pressão arterial, apenas $25 \%$ das unidades realizam a glicemia de jejum, 12,5\% realizam tratamento profilático para estrongiloidíase e nenhuma das unidades de saúde realizam tratamento profilático para osteoporose.

Em casos de neurite associada, nenhuma unidade de saúde realiza a imobilização do membro afetado com tala gessada ou encaminha o usuário para o ambulatório de ortopedia, $37,5 \%$ afirmam tomar outras condutas seguidas de orientação para repouso, $25 \%$ afirmam não tomar conduta nenhuma e 37,5\% afirmam nunca ter acompanhado nenhum caso relativo a esse tipo de reação.

Quanto à solicitação de exames diagnósticos complementares, identificou-se que $100 \%$ das unidades de saúde participantes da pesquisa solicitam exames de baciloscopia e 62,5\% solicitam histopatologia para hanseníase, sendo que, quando solicitados, ambos os exames são realizados no próprio município e posteriormente enviados para um laboratório conveniado de referência, sendo seu resultado disponibilizado ao usuário em média 30 dias após a coleta.

Dos usuários que apresentam reação tipo 
2, 75\% das unidades de saúde afirmam ofertar acompanhamento odontológico para esse público-alvo, enquanto $25 \%$ das unidades não oferecem esse tipo de atendimento como rotina no programa de hanseníase.

Entre os impressos utilizados no programa de hanseníase, $100 \%$ das unidades de saúde possuem a ficha de notificação de casos novos, o boletim de acompanhamento mensal dos casos atendidos, o formulário de avaliação do grau de incapacidade e o formulário para avaliação neurológica simplificada; $62,5 \%$ das unidades possuem o formulário de vigilância dos contatos intradomiciliares e $37,5 \%$ possuem a ficha de investigação de suspeita de recidiva.

Nenhuma das unidades de saúde do município possui vacina BCG disponível todos os dias da semana, sendo que, no município, tal vacina é disponibilizada apenas na sala de imunizações do hospital municipal.

Tabela 3. Solicitação de exames laboratoriais no diagnóstico da hanseníase e em casos de reações tipo 2. Canaã dos Carajás (PA) - 2016

\begin{tabular}{|c|c|c|c|c|c|c|}
\hline \multirow{3}{*}{ Exame Laboratorial } & \multicolumn{6}{|c|}{ Evento da Solicitação } \\
\hline & \multicolumn{3}{|c|}{ Diagnóstico } & \multicolumn{3}{|c|}{ Reação Tipo 2} \\
\hline & Sim & Não & Total & Sim & Não & Total \\
\hline Hemograma & $775 \%$ & $225 \%$ & $1100 \%$ & $550 \%$ & $550 \%$ & $1100 \%$ \\
\hline Creatinina & $225 \%$ & $775 \%$ & $1100 \%$ & $550 \%$ & $550 \%$ & $1100 \%$ \\
\hline TGO/TGP & $337,5 \%$ & $662,5 \%$ & $1100 \%$ & $550 \%$ & $550 \%$ & $1100 \%$ \\
\hline PCR & - & - & - & $225 \%$ & $775 \%$ & $1100 \%$ \\
\hline VHS/FAN & - & - & - & $112,5 \%$ & $887,5 \%$ & $1100 \%$ \\
\hline Urina & - & - & - & $550 \%$ & $550 \%$ & $1100 \%$ \\
\hline EPF & - & - & - & $225 \%$ & $775 \%$ & $1100 \%$ \\
\hline Outros & - & - & - & $112,5 \%$ & $887,5 \%$ & $1100 \%$ \\
\hline Nenhum & $225 \%$ & $775 \%$ & $1100 \%$ & $337,5 \%$ & $662,5 \%$ & $1100 \%$ \\
\hline
\end{tabular}

Fonte: Elaboração própria a partir dos dados coletados.

TGO: Transaminase Glutâmico Oxalacética; TGP: Transaminase Glutâmico Pirúvica; PCR: Proteína C Reativa; VHS: Velocidade de Hemossedimentação; FAN: Fator Antinuclear; EPF: Exame Parasitológico de Fezes.

Em relação aos exames laboratoriais solicitados no momento do diagnóstico do paciente com hanseníase, observa-se a partir da tabela 3 que não existe uma padronização específica para a solicitação de tais exames, sendo que o exame mais solicitado é o hemograma completo ( $75 \%$ das unidades) e o menos solicitado é a creatinina ( $25 \%$ das unidades). Todos os exames preconizados por intermédio do MS para o diagnóstico complementar ou controle dos casos de hanseníase e reações hansênicas são disponibilizados no município, observando-se assim um não cumprimento das normas estabelecidas para o programa e questão.

Nos casos de reação tipo 2, também se observa uma falta de padronização no acompanhamento de indicadores laboratoriais em que $37,5 \%$ das unidades de saúde não solicitam nenhum exame; $50 \%$ delas solicitam hemograma, creatininina, Transaminase Glutâmico Oxalacética/Transaminase Glutâmico Pirúvica (TGO/TGP) e urina e apenas $25 \%$ das unidades solicitam Exame Parasitológico de Fezes (EPF) e Proteína C Reativa (PCR). 
Tabela 4. Materiais disponibilizados ao usuário do programa municipal de controle da hanseníase de Canaã dos Carajás (PA) - 2016

\begin{tabular}{lrrr}
\hline \multirow{2}{*}{ Material } & \multicolumn{3}{c}{ Disponibilidade } \\
\cline { 2 - 4 } & \multicolumn{1}{c}{ Sim } & Não & Total \\
\hline Colírio para reposição de lágrima & $12,5 \%$ & $87,5 \%$ & $100 \%$ \\
Soro fisiológico & $100 \%$ & $0 \%$ & $100 \%$ \\
Óleo de girassol (Dersane) & $37,5 \%$ & $62,5 \%$ & $100 \%$ \\
Óleo mineral & $75 \%$ & $25 \%$ & $100 \%$ \\
Creme de ureia & $25 \%$ & $75 \%$ & $100 \%$ \\
Outros & $12,5 \%$ & $87,5 \%$ & $100 \%$ \\
\hline
\end{tabular}

Fonte: Elaboração própria a partir dos dados coletados.

Das unidades de saúde participantes da pesquisa, $100 \%$ disponibilizam gratuitamente soro fisiológico aos pacientes com hanseníase; $75 \%$ distribuem óleo mineral; 37,5\% disponibilizam óleo de girassol; $25 \%$ disponibilizam creme de ureia e $12,5 \%$ disponibilizam colírio para reposição de lágrima, conforme especificado na tabela 4.

Nenhuma das unidades de saúde abre sábado ou domingo ou permanece aberta no horário de almoço ou após as $18 \mathrm{~h}$ algum dia na semana.

$\mathrm{Na}$ opinião do gestor do programa, o município possui como pontos fortes (forças) a pouca rotatividade da equipe de saúde, a disponibilidade de materiais e insumos para o programa e o apoio técnico e proximidade da referência para os atendimentos mais complexos. Possui como pontos fracos (fraquezas) a mobilidade populacional, a dificuldade para a busca ativa de contatos e a frequente entrada de novos servidores no quadro, devido à expansão populacional e dos serviços de saúde e a necessidade de capacitação frequente dessa nova demanda de profissionais.

\section{Discussão}

A presença de profissionais de diversas categorias é de extrema importância na assistência da hanseníase, tendo em vista a necessidade da prestação de cuidados de forma integral a esse grupo de usuários. A presença da equipe multiprofissional propicia um atendimento acolhedor e uma maior resolutividade do trabalho, permitindo um aprofundamento de saberes e práticas e a geração de vínculos comunitários, gerando uma maior autonomia no processo de trabalho e assistência da atenção primária (COSTA ET AL., 2014).

A ausência do profissional de terapia ocupacional na equipe de trabalho atuante na hanseníase traz prejuízos direto ao paciente. Embora Canaã dos Carajás seja um município com população abaixo de 100 mil habitantes, e possa pactuar tal serviço com outros municípios, seria de extrema importância a contratação desse profissional para atuar nas ações de reabilitação em hanseníase.

A terapia ocupacional no cuidado ao paciente com hanseníase é fundamental para a compreensão do usuário enquanto sujeito de sua própria história, atuando por intermédio da reabilitação física direcionada às atividades da vida diária, por meio da mudança e da transformação do indivíduo, na promoção do autocuidado e da independência desse usuário (LOUREIRO; BARRETO; MAKSUD, 2015).

Para a OMS (2010), os serviços de atendimento à hanseníase não necessitam de grandes aparatos tecnológicos, devendo proporcionar uma ampla cobertura, sendo 
fornecido em todas as unidades de saúde, centrado no paciente garantindo privacidade e confidencialidade, possuir diagnóstico oportuno e os recursos necessários e esse fim, tratamento disponível e gratuito e garantir encaminhamentos adequados em casos de complicações, reabilitação e outras situações que requeiram um serviço especializado.

Diante da complexidade da hanseníase, de seu histórico de estigma e segregação e de todo o contexto social que envolve a doença, faz-se necessária a elaboração de medidas específicas que visam minimizar os efeitos da doença na comunidade, bem como ampliar as taxas de detecção de casos novos para posteriormente controlar a doença por meio de medidas que interrompam sua cadeia de transmissão. Assim, a elaboração do plano municipal de controle da hanseníase é uma medida simples e inovadora capaz de direcionar as ações da doença de acordo com seus níveis de complexidade e de atenção, impactando diretamente nos coeficientes e indicadores da doença, uma vez que dará mais visibilidade às ações e serviços que precisam ser desenvolvidos para esse fim.

No município da pesquisa, foi realizada apenas uma campanha para hanseníase. As campanhas para detecção de casos novos de hanseníase devem ser vistas como uma ferramenta facilitadora do processo de trabalho e que agrega grandes contribuições para melhoria dos coeficientes da doença. Em um estudo realizado no município de Buriticupu, estado do Maranhão, os autores identificaram que a busca ativa de casos novos é de extrema importância para o diagnóstico precoce da doença (SILVA ET AL., 2010).

A baixa taxa de capacitação profissional no município de Canaã dos Carajás é um fator preocupante no que se refere a prestação de assistência em hanseníase. Observou-se que o município ofereceu, no último ano, treinamento apenas para os profissionais de nível superior, não atendendo às demandas de outros profissionais como técnico/auxiliar de enfermagem e agentes comunitários de saúde.
Um estudo realizado em Minas Gerais identificou também uma baixa taxa de treinamentos e capacitações ofertadas aos profissionais que atuam no atendimento da hanseníase nas unidades de APS. Diante de tal fato, a autora sugere a adoção de mecanismo de qualificação a distância e consultorias por parte de municípios que não possuem profissionais capacitados para ministrar tais treinamentos (LANZA, 2014).

$O$ uso de recursos financeiros municipais como contrapartida para a aquisição de insumos e outros materiais para a manutenção do programa municipal de controle da hanseníase é uma ação prevista e rotineira que, teoricamente, deve existir em todos os municípios brasileiros, tendo em vista o caráter tripartite de financiamento da saúde, assegurado por intermédio de lei federal (BRASIL, 1990).

A falta de uma programação orçamentária e financeira específica para a hanseníase, no contexto da lei orçamentaria anual, não deve prejudicar o desenvolvimento das ações específicas para esse agravo, uma vez que o próprio MS determina os repasses financeiros alocados em blocos de financiamento, em que a hanseníase e outras doenças transmissíveis pertencem ao conjunto das ações e financiamento do bloco de vigilância em saúde (BRASIL, 2016).

O serviço de saúde usa o cartão de aprazamento das consultas, inclusive para as referências para o serviço especializado, conforme preconiza a Portaria MS $n^{\circ}$ 149/2016 (BRASIL 2016).

A busca ativa de pacientes faltosos no programa de hanseníase e de contatos domiciliares é de extrema importância para o resgate da demanda que não compareceu a unidade de saúde e requer assistência profissional. Canaã dos Carajás possui ações inovadoras como o fornecimento de combustível para os profissionais das unidades de saúde realizarem tais visitas em seu próprio veículo, caso não optem por usar o veículo disponibilizado pelo serviço. 
Um estudo realizado em Goiás identificou que entre os motivos para o não comparecimento à unidade de saúde na data agendada está a não informação sobre o agendamento realizado e a distância da residência até o serviço de saúde limitando o acesso do usuário (FARIA ET AL., 2013).

$\mathrm{O}$ uso de protocolos municipais e fluxogramas de atendimento no programa de hanseníase é de extrema importância para unificação da assistência prestada ao paciente, dentro de um contexto de equidade, regionalização e valorização da singularização das características locais, bem como de seus condicionantes e determinantes. O protocolo deve ser visto como um instrumento técnico de orientação profissional e do processo de trabalho, por meio do direcionamento das atividades a serem desempenhadas dentro das atribuições profissionais de cada categoria dentro do serviço de saúde, com o objetivo de garantir melhoria na qualidade da assistência prestada ao usuário (COREN-MG, 2012).

Referente ao uso de materiais educativos como folder e cartaz, percebeu-se que a maioria das unidades de saúde faz uso de tais recursos informativos e educativos. Em um estudo realizado no Rio de Janeiro sobre o uso de recursos para comunicação, os autores concluíram que tais materiais educativos favorecem a compreensão de aspectos clínicos, psicológicos e socioculturais sobre a hanseníase, potencializando o diálogo entre os grupos (SANTOS; RIBEIRO; MONTEIRO, 2012).

O município dispõe de um kit com os materiais necessários à realização do diagnóstico da hanseníase comportando: tubo de ensaio, estesiômetro, fio dental sem sabor, alfinetes e algodão. Todos esses materiais são utilizados para avaliar o grau de incapacidade e a ausência ou diminuição de sensibilidade, conforme disposto pelo MS (BRASIL, 2016).

Quanto à realização do teste de acuidade visual no diagnóstico, a maioria das unidades de saúde (75\%) não realiza tal teste por falta de material na unidade de saúde e por falta também de um espaço adequado para tal procedimento. Em uma avaliação realizada em um centro de referência nacional para hanseníase, identificou-se que houve aumento no número de deficiências ou incapacidades oculares comparados no momento do diagnóstico e na alta por cura respectivamente. Os autores afirmam que tal fato se torna recorrente devido ao pequeno número de serviços em que a avaliação ocular faz parte da rotina de controle dos pacientes portadores de hanseníase (KIL ET AL., 2010).

Referente ao armazenamento da Poliquimioterapia (PQT) para hanseníase, identificou-se que apenas $50 \%$ das unidades de saúde possuem armazenamento das medicações no próprio estabelecimento, enquanto as demais unidades fazem a solicitação mediante o diagnóstico do paciente. Vale atentar para o fato de que o armazenamento da medicação na própria unidade favorece a dispensação imediata, sem atrasos no início da PQT e sem riscos de perda do paciente ou ausência caso ocorra agendamento para dispensação da medicação para o próximo dia.

O MS define que o uso do cartão de aprazamento ou agendamento de consultas é de extrema importância para o registro da data de retorno ao serviço de saúde, servindo como ferramenta para monitorar a adesão do paciente ao tratamento. Em Canaã dos Carajás, todas as unidades participantes da pesquisa fazem uso de tal dispositivo no programa de hanseníase (BRASIL, 2016).

Os corticosteroides, neste caso a prednisona, trazem grandes benefícios ao estado de saúde do usuário, porém seus efeitos colaterais devem ser monitorados perante o risco de comorbidades, agravamento de doenças preexistentes e desencadeamento de efeitos danosos. Observou-se que as unidades de saúde de Canaã dos Carajás, que participaram da pesquisa, não possuem uma padronização para o monitoramento de ações implantadas no paciente em uso desse medicamento, sendo que a maioria das unidades realiza somente o peso e a pressão arterial, sendo esporádico o monitoramento da 
glicemia e os cuidados com estrongiloidíase e osteoporose, conforme preconização do MS (BRASIL, 2016; RIO DE JANEIRO, 2010),

Observou-se que as unidades de saúde não estão preparadas para atender ou dar o encaminhamento necessário aos casos de neurite associada, sendo que nenhuma das unidades pesquisada orienta o repouso do membro por meio de imobilização com tala gessada (BRASIL, 2016).

Identificou-se que a maioria das unidades de saúde do município pesquisado faz uso da baciloscopia e da histopatologia como recursos auxiliares no diagnóstico da hanseníase, sendo tal serviço oferecido gratuitamente aos usuários que necessitam. Para Obardia, Veraldino e Alves (2011), tais exames são úteis no diagnóstico classificatório da doença em situações em que o diagnóstico clínico suscitou dúvidas, porém não devem ser considerados como exames de escolha para o diagnóstico da doença, devendo as características clínicas prevalecerem sempre.

$\mathrm{O}$ atendimento à saúde bucal dos pacientes de hanseníase está presente na maioria das unidades de saúde que participaram do estudo (75\%). Isso demonstra comprometimento com a integralidade da assistência em saúde prestada ao usuário desse programa. Um estudo sobre a autopercepção sobre saúde bucal com pacientes de hanseníase demonstrou que é de extrema importância o desenvolvimento de programas que promovam a saúde bucal em hanseníase uma vez que infecções odontológicas podem causar reações hansênicas, agravando assim os sintomas da doença (ALMEIDA ET AL, 2013).

O prontuário completo e organizado é de extrema importância para situar os diversos profissionais que fazem atendimento ao usuário de hanseníase sobre todas as situações, condutas e assistência prestada. O prontuário com anotações adequadas e os anexos inerentes ao estado de saúde do paciente facilitam o acesso ao histórico daquele usuário, permitindo ao profissional consultar outras intervenções já adotadas e assim planejar melhor sua conduta diante do problema atual (PASCHOAL ET AL., 2011).

A ausência de vacina BCG nas unidades da rede de atenção primária é um fator agravante que precisa ser solucionado a fim de melhorar o atendimento aos contatos dos pacientes de hanseníase. O que se observa é que tal problema não é restrito a Canaã dos Carajás e que vários municípios do País estão com falta desse imunobiológico em sua rede de frios, sendo necessário o agendamento de dias para realizar a vacina e até mesmo a centralização desta em apenas um local. Esse problema ocorre devido a atrasos no repasse dessa vacina ou do repasse em quantidade inferior à necessidade de estados e municípios, não sendo possível atender toda a demanda de pessoas que necessitam desse imunobiológico segundo as normas do programa municipal de imunização (CANCIA, 2015).

Quanto aos exames laboratoriais solicitados no momento do diagnóstico e em casos de reações hansênicas, observa-se que não existe uma padronização ou protocolo seguido pelas unidades de saúde, sendo que algumas solicitam todos os exames e outras solicitam parcialmente, porém sem critérios específicos para isso, demonstrando que, nesse quesito, o município não está de acordo com o que preconiza o MS (BRASIL, 2016).

A disponibilização gratuita de insumos ao usuário de hanseníase não segue o mesmo padrão em todas as unidades. Todas as unidades dispõem de soro fisiológico, mas apenas $12,5 \%$ disponibilizam colírio para reposição de lágrimas. O ideal seria a oferta dos insumos necessário ao atendimento do paciente em todas as unidades de saúde do município, conforme a demanda de cada serviço. A oferta de insumos e materiais ao usuário do serviço, mesmo que este não esteja previsto no elenco de medicamentos básicos, é de responsabilidade das unidades de saúde, por meio de disponibilização pelo setor específico, favorecendo, assim, o exercício da integralidade da atenção e do serviço em questão (SÃO PAULO, 2013; RIO DE JANEIRO, 2010). 
O fato de nenhuma unidade de saúde ficar aberta após as $18 \mathrm{~h}$, no horário de almoço ou no sábado ou domingo não prejudica diretamente o desenvolvimento das ações de hanseníase, mas dificulta o acesso dos usuários que trabalham em turnos, como é o caso da maioria dos trabalhadores do município em decorrência dos horários estabelecidos pelas empresas de mineração e suas prestadoras de serviço. Tal fato requer que, nestes casos de trabalho em regime de escala ou turnos, o usuário necessite faltar ao serviço para ser atendido na unidade de saúde.

Um estudo realizado no município de João Pessoa (PB), com o objetivo de analisar o impacto da ampliação do horário de funcionamento das unidades de saúde para o turno noturno, identificou uma melhora significativa da procura pelo serviço principalmente da clientela de trabalhadores masculinos. Tais resultados apontam para uma potencial eficácia da criação de horários alternativos para o atendimento principalmente de trabalhadores que não podem procurar o serviço de saúde nos horários padrões de funcionamento (CORDEIRO ET AL., 2014).

No que se refere aos pontos fortes e pontos fracos do programa de hanseníase do município, segundo a opinião do gestor do sistema de saúde, observa-se que as forças estão mais relacionadas com a gestão de pessoas e orçamentária e que as fraquezas estão relacionadas com as dificuldades impostas pelo meio.

Quanto à mobilidade populacional, destacada como ponto fraco do programa em questão, um estudo realizado em Mato Grosso identificou uma associação entre a evolução e aumento no número de casos de hanseníase e o processo de ocupação do território. A autora destaca ainda que a migração explicaria a instalação e evolução da hanseníase, porém a manutenção dela estaria relacionada com determinantes e condicionantes fornecidos pelo meio, como o aumento de desmatamento e a expansão do extrativismo e da agropecuária (MAGALHÃES ET AL., 2011).

Este estudo possui como limitações o tamanho amostral utilizado, embora a amostra empregada tenha representado 92,3\% do universo disponível no município. Destaca-se também a ausência de um instrumento de coleta de dados validado para a avaliação de estrutura, capaz de indicar parâmetros avaliatórios eficazes e que ultrapassem os limites atuais encontrados na subjetividade da análise realizada.

\section{Conclusões}

Observou-se, a partir deste estudo, que o município de Canaã dos Carajás, com base na Portaria GM/MS n 149/2016 que define as diretrizes para vigilância, atenção e controle da hanseníase, possui uma estrutura que, na maioria dos itens avaliados, encontra-se classificada entre insatisfatória e regular, sendo poucos os itens avaliados que receberam classificação bom ou excelente. Foram encontradas diversas fragilidades no que tange às orientações dadas pela portaria acima citada e que precisam ser revistas no intuito de garantir melhoria na qualidade da assistência prestada ao usuário.

É necessário que o município em questão fomente maiores investimentos na capacitação profissional e educação permanente dos trabalhadores ligados à hanseníase a fim de que estes sintam maior segurança no atendimento ao usuário desse programa e que isto repercuta diretamente na qualidade da assistência prestada, implementando mecanismos de vigilância epidemiológica para o agravo em questão, fortalecendo o seu programa de controle, promovendo melhorias nos processos de assistência e do resultado final do programa.

Sugere-se a realização de novos estudos, com os mesmos seguimentos, porém com uma amostragem maior, a fim de que se possa comparar os resultados obtidos e, a partir destes dados, formular hipóteses para as questões encontradas e levantadas durante a avaliação. 


\section{Referências}

ALMEIDA, J. R. S. et al. Autopercepção de pessoas acometidas pela hanseníase sobre sua saúde bucal e necessidade de tratamento. Ciênc. saúde coletiva, Rio de Janeiro, v. 18, n. 3, p. 817-826, mar. 2013.

BRASIL. Lei no 8.080, de 19 de setembro de 1990. Dispõe sobre as condições para a promoção, proteção e recuperação da saúde, a organização e o funcionamento dos serviços correspondentes e dá outras providências. Diário Oficial [da] República Federativa do Brasil, Brasília, DF, 20 set. 1990. Disponível em: <https://www. google.com.br/?gfe_rd=cr\&ei=IdLUVtalIfTM8AfCob2o BA\#>. Acesso em: 13 jan. 2016.

Ministério da Saúde. Indicadores e dados básicos Brasil: indicadores de morbidade 2012. Disponível em: <http://tabnet.datasus.gov.br/cgi/idb2012/matriz. htm\#morb>. Acesso em: 20 out. 2016.

Ministério da Saúde. Portaria ${ }^{\circ}$ 149, de 3 de fevereiro de 2016. Aprova as diretrizes para Vigilância, Atenção e Eliminação da Hanseníase como Problema de Saúde Pública, com a finalidade de orientar os gestores e os profissionais dos serviços de saúde. Diário Oficial [da] União, Brasília, DF, 4 fev. 2016. Disponível em: <http://www.cve.saude.sp.gov.br/htm/hans/ pdf/U_PT-MS-GM-149_030216.pdf.>. Acesso em: 4 fev. 2016.

Ministério da Saúde. Secretaria de Atenção em Saúde. Departamento de Atenção Básica. Informe da atenção básica $n^{\circ}$ 42. Brasília, DF: Ministério da Saúde, 2007. Disponível em: <http://bvsms.saude.gov.br/bvs/ publicacoes/07_1120_P.pdf>. Acesso em: 20 out. 2016.

CANCIAN, N. Falta de vacinas atinge postos de saúde de ao menos seis estados. Folha de S. Paulo, Brasília, DF,18 mar. 2015. Disponível em: <http://wwwl.folha. uol.com.br/cotidiano/2015/03/1604398-falta-de-vacinas-atinge-postos-de-saudes-de-ao-menos-seis-estados.shtml>. Acesso em: 2 fev. 2016.

CONSELHO REGIONAL DE ENFERMAGEM DE MINAS GERAIS (COREN-MG). Guia para elaboração de protocolo assistencial de enfermagem para a atenção básica. Belo Horizonte: Coren-MG, 2012. Disponível em: <https://www.google.com.br/?gfe_rd=cr\&ei=IdLU VtalIfTM8AfCob2oBA\#>. Acesso em: 31 jan. 2016.

CORDEIRO, S. V. L. et al. Atenção básica à saúde masculina: possibilidades e limites no atendimento noturno. Esc. Anna Nery, Rio de Janeiro, v. 18, n. 4, p. 644-649, dez. 2014. Disponível em: <http://www. scielo.br/scielo.php?script=sci_arttext\&pid=S1414$-81452014000400644 \& \operatorname{lng}=e n \& n r m=$ iso $>$. Acesso em: 27 fev. 2016

COSTA, J. P. et al. Resolubilidade do cuidado na atenção primária: articulação multiprofissional e rede de serviços. Saúde debate, Rio de Janeiro, v. 38, n. 103, p. 733-743, dez. 2014.

DONABEDIAN, A. Explorations in quality assesment and monitoring. v. 1. Michigan: Health Admnistration Press, 1980.

FARIA, D. R. et al. Avaliação de Contatos de Hanseníase. Revista Panorâmica On-Line. Barra do Garças, v. 14, p. 35-46, jul. 2013.

FERREIRA, F. X. Análise da implantação do programa de eliminação da hanseníase em Manaus. 2005. 133 f. Dissertação (Mestrado em Ciências na Área de Saúde Pública) - Escola Nacional de Saúde Pública Sergio Arouca, Fundação Oswaldo Cruz, Manaus, 2005.

GIL, A. C. Métodos e técnicas de pesquisa social. 6. ed. São Paulo: Atlas, 2008.

KIL, A. K. A. et al. Deficiências e Incapacidades por Hanseníase: avaliação clínica e epidemiológica dos pacientes atendidos em um Centro de Referência Nacional do Brasil. Hansen Int, Bauru, v. 37, n. 1, p. 2533, 2012.

LANZA, F. M. Avaliação da atenção primária no controle da hanseníase: validação de instrumentos e análise do desempenho de municípios endêmicos do Estado de Minas Gerais. 2014. Tese. (Doutorado em Enfermagem) - Escola de Enfermagem, Universidade Federal de 
Minas Gerais, Belo Horizonte, 2014.

LOUREIRO, L. A.; BARRETO, L. L.; MAKSUD, I.

Percepções sobre a terapia ocupacional no cuidado ao paciente com hanseníase. REFACS, Uberaba, v. 3, sup.1, p. 134-141, 2015.

MAGALHÃES, M. C. C. et al. Migração e hanseníase em Mato Grosso. Rev Bras Epidemiol., São Paulo, v. 14, n. 3, p. 386-97, set. 2011.

OBARDIA, D. L. L.; VERARDINO, G.; ALVES, M. F. G. S. Hanseníase: correlação clínico-histopatológica. Revista Hospital Universitário Pedro Ernesto, v. 10, n. 1, p. 20-23, jan./mar. 2011.

ORGANIZAÇÃO MUNDIAL DE SAÚDE (OMS). Estratégia global aprimorada para redução adicional da carga da hanseníase: período do plano: 2011-2015. Brasília, DF: Organização Pan-Americana de Saúde, 2010. Disponível em: <http://bvsms.saude.gov.br/bvs/ publicacoes/estrategia_global_aprimorada_reducao_ hanseniase.pdf>. Acesso em: 20 out. 2016.

PASCHOAL, V. D. A. et al. Criação de banco de dados para sustentação da pós-eliminação em hanseníase. Ciênc. saúde coletiva, Rio de Janeiro, v. 16, supl. 1, p. 1201-1210, 2011.

RIO DE JANEIRO (Município). Secretaria Municipal de Saúde e Defesa Civil. Sub-Secretaria de Promoção, Atenção Primária e Vigilância em Saúde. Linha de Cuidado da Hanseníase. Rio de Janeiro, 2010. Disponível em: <http://www.ensp.fiocruz.br/portal-ensp/judicializacao/pdfs/487.pdf $>$. Acesso em: 2 fev. 2016.

SANTOS, A. K.; RIBEIRO, A. P. G.; MONTEIRO, S. Hansen's disease and communication practices: study on the reception of educational materials at a healthcare clinic in Rio de Janeiro. Interface, Botucatu, v. 16, n. 40, p. 205-218, jan./mar. 2012.

SÃO PAULO (Prefeitura). Secretaria Municipal de Saúde. Protocolo para o atendimento nas unidades de referência para hanseníase. São Paulo, 2013. Disponível em: <http://www.prefeitura.sp.gov.br/cidade/secretarias/upload/chamadas/protocolo_hanseniase_2013_final_1380575286.pdf>. Acesso em: 10 fev. 2016.

SARNO, E. N. A hanseníase no laboratório. História, Ciência e Saúde, Rio de janeiro, v. 10, supl. 1, p. 277-290, 2003.

SILVA, A. R. et al. Hanseníase no Município de Buriticupu, Estado do Maranhão: busca ativa de casos na população adulta. Rev. Soc, Bras. Med. Trop., Uberaba, v. 43. n. 6, p. 691-694, nov./dez. 2010.

\footnotetext{
Recebido para publicação em março de 2016

Versão final em agosto de 2016

Conflito de interesses: inexistente

Suporte financeiro: não houve
} 